\title{
Profesorado morto por mor da represión franquista
}

Presentamos a relación de profesorado morto como consecuencia da represión practicada polo franquismo. Suman en total 100 persoas, das cales 98 son homes e dúas mulleres: Mercedes Romero Abella e María Vázquez Suárez.

A maioría, en concreto 77, eran mestres e mestras que estaban á fronte dunha escola pública en xullo de 1936; rexístrase o seu nome, a idade e a escola en que exercían, así como a modalidade de morte que padeceron, o lugar en que se produciu e o ano. Entre os 23 restantes, 17 tamén eran mestres, pero non exercían en Galicia, estaban en excedencia ou ben traballaban no ensino privado ou noutras ocupacións; un era profesor de Maxisterio e outro alumno normalista, e só catro carecían de relación co ensino primario. Existe constancia dalgunhas outras mortes, aínda que non as incluímos por seren moi escasas e imprecisas as referencias de que se dispón.

Pertencían á provincia da Coruña —ou morreron nela- 39 persoas, 30 a Pontevedra, 16 a Ourense e 15 a Lugo. A idade media para o conxunto de Galicia era de algo máis de 35 anos.

Foron “paseados" 48 profesores e dúas profesoras e executados 38, rexistrándose un equilibrio entre ambas modalidades en Lugo, un predominio das execucións en Pontevedra e moitos máis "paseos" que execucións na Coruña, e sobre todo en Ourense, cando menos en termos relativos. Catro persoas morreron no cárcere, tres por mor das malleiras sufridas, dúas suicidáronse e de tres carecemos de información.

As máis das mortes con data coñecida, concretamente 62, concentráronse en 1936, 21 tiveron lugar en 1937, 4 en 1938, 2 en 1939, 2 en 1940 e 1 en 1941. Así pois, o 67\% producíronse no segundo semestre de 1936, e o $90 \%$ antes de se iniciar 1938. Con todo, en anos sucesivos aínda seguirán rexistrándose mortes, coma a de José Gómez Gayoso, secretario do Partido Comunista en Galicia, a quen se lle aplicou o garrote vil en 1948.

A listaxe que presentamos está elaborada a partir das investigacións realizadas polos historiadores e historiadoras que traballan neste campo, algúns de cuxos resultados se condensan na base de datos Nomes e Voces. Somos conscientes de que non é exhaustiva, e de que algúns datos non son todo o fiables que nos gustaría, polo que pedimos desculpas. En todo caso, aspiramos a que entre todos e todas poidamos ir completando esta relación, que á súa vez dá continuidade a outras. 


\section{A CORUÑA}

\section{Maxisterio público en exercicio na provincia en xullo de 1936}

\begin{tabular}{|c|c|c|c|c|c|c|}
\hline \multirow{2}{*}{ Nome } & \multirow{2}{*}{ Idade } & \multirow{2}{*}{ Escola onde exercía } & \multicolumn{3}{|c|}{ Morte } & \multirow{2}{*}{ Ano } \\
\hline & & & $\mathbf{P}$ & E & Concello & \\
\hline Pedro Almazán Ramos & 27 & O Val (Narón) & $x$ & & Ferrol & 1936 \\
\hline Enrique Alonso Teijeiro & 23 & Cedeira & & $\mathrm{X}$ & Ferrol & 1936 \\
\hline Rodrigo Alvárez Gantes & 30 & Escarabote (Boiro) & & $\mathrm{X}$ & Santiago & 1937 \\
\hline José Ambrós Gordillo & 36 & Vilarrube (Valdoviño) & $\mathrm{x}$ & & Ferrol & 1936 \\
\hline Fernando Barcia Beiras & 52 & Ameneiro (Teo) & & $x$ & Santiago & 1938 \\
\hline Angel Caamaño Villaverde & 27 & Toba (Cee) & & $\mathrm{X}$ & Santiago & 1937 \\
\hline José Caballero Rubio & & Abanqueiro (Boiro) & $\mathrm{x}$ & & Ponteareas & 1936 \\
\hline José R. Fernández Barral & 37 & Pontedeume & $x$ & & Ares & 1936 \\
\hline Rafael Fernández Casas & 34 & Souto (Sada) & $\mathrm{x}$ & & Cesuras & 1936 \\
\hline Ramón A. Figueroa Vilachá & 33 & Sésamo (Culleredo) & & $\mathrm{X}$ & A Coruña & 1940 \\
\hline José García Fernández & 42 & Ordes & & $\mathrm{X}$ & Santiago & 1937 \\
\hline Juan García Niebla & 60 & A Graña (Ferrol) & $\mathrm{x}$ & & Ferrol & 1936 \\
\hline Julián Hermida Montoya & 29 & A Coruña & $\mathrm{x}$ & & Carral & 1936 \\
\hline Jesús Álvaro López Brenlla & 24 & Louro (Muros) & $x$ & & Muros & 1936 \\
\hline Manuel López González & 26 & Atios (Valdoviño) & $\mathrm{x}$ & & Ferrol & 1936 \\
\hline Pedro López Vázquez & 44 & San Mamede (Carnota) & \multicolumn{2}{|c|}{ Malleira } & Carnota & 1937 \\
\hline José Losada Castelao & 45 & Rianxo & \multicolumn{2}{|c|}{ Suicidio } & Vigo & 1937 \\
\hline Bernardo Mato Castro & 49 & Teo & \multicolumn{2}{|c|}{ Malleira } & A Estrada & 1936 \\
\hline Eugenio Meizoso Nuñez & 28 & Sedes (Narón) & $\mathrm{x}$ & & Narón & 1936 \\
\hline José Novás Soto & 29 & San Clodio (Ortigueira) & $\mathrm{x}$ & & Ferrol & 1936 \\
\hline Rafael Pardo Carmona & 37 & Serres (Muros) & & $\mathrm{X}$ & Santiago & 1936 \\
\hline Rufino Redondo Senra & 24 & Devesos (Cerdido) & & $\mathrm{X}$ & Ferrol & 1936 \\
\hline Manuel Rodríguez Castelao & 31 & O Pazo (Rianxo) & \multicolumn{2}{|c|}{ Suicidio } & Vigo & 1937 \\
\hline Mercedes Romero Abella & 29 & A Coruña & $\mathrm{x}$ & & Aranga & 1936 \\
\hline Manuel Santeiro Boo & 33 & Ortigueira & $\mathrm{X}$ & & A Coruña & 1936 \\
\hline Reinaldo Sarria Delgado & 34 & Aviño (Valdoviño) & \multicolumn{2}{|c|}{ Malleira } & Valdoviño (?) & 1941 \\
\hline
\end{tabular}




\begin{tabular}{l|c|l|l|l|l|l|l}
\hline María Vázquez Suárez & 33 & Miño & X & & \multicolumn{1}{l}{ Miño } & 1936 \\
\hline \multicolumn{7}{|c|}{ Outro profesorado } \\
\hline Eugenio Carré Naya & 20 & $\begin{array}{l}\text { Mestre (Secretario de } \\
\text { FETE-UGT) }\end{array}$ & X & & A Coruña & 1936 \\
\hline Francisco Gómez Blanco & 23 & Mestre (natural da Coruña) & X & & Culleredo & 1936 \\
\hline Andrés González Guianes & 28 & Mestre (natural de Santiago) & X & & Teo & 1936 \\
\hline Leonardo Martínez Novo & 25 & $\begin{array}{l}\text { Mestre (Col. Curros Enríquez, } \\
\text { Pontedeume) }\end{array}$ & X & & Pontedeume & 1936 \\
\hline Manuel de Pazos Fernández & 27 & Mestre (natural de Ferrol) & X & & Ferrol & 1936 \\
\hline Vicencio Prieto Ramos & 24 & Mestre (natural de León) & & X & Ferrol & 1937 \\
\hline Ramón Tajes Lago & 27 & Mestre (natural de Muros) & & X & Asturias & 1938 \\
\hline Luís Rastrollo González & 28 & Normalista (Santiago) & & X & Santiago & 1936 \\
\hline Manuel Rodríguez Méndez & & Mestre & X & & Ferrol & 1936 \\
\hline Leandro Carré Brandaríz & & Escola de Traballo (A Coruña) & & & Asturias & 1937 \\
\hline Gerardo Abad Conde & 55 & $\begin{array}{l}\text { Escola de Comercio } \\
\text { (A Coruña) }\end{array}$ & X & & Madrid & 1936 \\
\hline Luís Rufilanchas Salcedo & 27 & Universidade (Madrid) & & X & A Coruña & 1937 \\
\hline
\end{tabular}

LUGO

\begin{tabular}{|c|c|c|c|c|c|c|}
\hline \multicolumn{7}{|c|}{ Maxisterio público en exercicio na provincia en xullo de 1936} \\
\hline \multirow{2}{*}{ Nome } & \multirow{2}{*}{ Idade } & \multirow{2}{*}{ Escola onde exercía } & \multicolumn{3}{|c|}{ Morte } & \multirow{2}{*}{ Ano } \\
\hline & & & $\mathbf{P}$ & $\mathbf{E}$ & Concello & \\
\hline Juan Abramo Dios & 28 & A Igrexa (A Pobra do Brollón) & $x$ & & Pobra Brollón & 1936 \\
\hline $\begin{array}{l}\text { Hermenegildo Alonso } \\
\text { Sánchez }\end{array}$ & 38 & Vilaoudríz (A Pontenova) & $\mathrm{x}$ & & A Pontenova & 1939 \\
\hline Ramón Bou Gil & 37 & Castillón (Ferreira de Pantón) & $x$ & & Monforte & 1936 \\
\hline José Costa Pardo & 29 & $\begin{array}{l}\text { San Román-Vilaestrofe } \\
\text { (Cervo) }\end{array}$ & \multicolumn{2}{|c|}{ Cárcere } & Lugo & 1939 \\
\hline Angel Fraga Orosa & 38 & Vieiro (Viveiro) & & $\mathrm{x}$ & Lugo & 1936 \\
\hline José María Gallo y Gallo & 25 & A Pontenova & & $x$ & Lugo & 1937 \\
\hline David Labrador Zuil & 35 & Vilaoudriz (A Pontenova) & & $x$ & Lugo & 1936 \\
\hline Antonio Lafuente Jiménez & 27 & Muras & & $x$ & Lugo & 1937 \\
\hline César Margolles de la Vega & & Benquerencia (Barreiros) & $x$ & & Asturias & \\
\hline Emilio Muiños Guerra & 50 & Vilacaíz (O Saviñao) & $x$ & & Paradela & 1937 \\
\hline
\end{tabular}




\begin{tabular}{l|l|l|l|l|l|l}
\hline Antonio Palmeiro & 51 & Luaces (Pol) & X & & & \\
\hline Evaristo Pereira Rubio & 61 & Sarria & & X & Lugo & 1936 \\
\hline José Ramos López & 25 & Lugo (?) & & X & Lugo & 1937 \\
\hline Arximiro Rico Trabada & 32 & San Bernabel (Baleira) & X & & Castroverde & 1937 \\
\hline \multicolumn{7}{c}{ Outro profesorado } \\
\hline Ignacio Álvarez Álvarez & 30 & Mestre (Navia de Luarca) & & X & Lugo & 1936 \\
\hline \multicolumn{7}{c}{ OURENSE }
\end{tabular}

\begin{tabular}{|c|c|c|c|c|c|c|}
\hline \multicolumn{7}{|c|}{ Maxisterio público en exercicio na provincia en xullo de 1936} \\
\hline \multirow{2}{*}{ Nome } & \multirow{2}{*}{ Idade } & \multirow{2}{*}{ Escola onde exercía } & \multicolumn{3}{|c|}{ Morte } & \multirow{2}{*}{ Ano } \\
\hline & & & $\mathbf{P}$ & E & Concello & \\
\hline Rafael Alonso Rodríguez & 34 & Amoeiro & $x$ & & Maceda & 1937 \\
\hline José L. Bouzas Fernández & 30 & Flariz (Monterrei) & $x$ & & A Gudiña & 1936 \\
\hline Antonio Caneda Rodríguez & 31 & Medos (S. Xoán de Río) & $x$ & & O Irixo & 1937 \\
\hline Antonio Dopazo Fernández & 27 & Tirós (Pereiro de Aguiar) & $x$ & & Ribadavia & 1936 \\
\hline Julio González Álvarez & 25 & Gontán (Verea) & $x$ & & Verea & 1936 \\
\hline Amadeo López Bello & 32 & Beiro (Carballeda de Avia) & $x$ & & Verea & 1936 \\
\hline Eligio Núñez Muñoz & 30 & Cambeo (Coles) & $x$ & & Montederramo & 1936 \\
\hline Camilo Palmeiro Fernández & & Corzos (A Veiga) & $x$ & & A P. de Trives & \\
\hline Justo Prada López & 55 & $\begin{array}{l}\text { Córgomo } \\
\text { (Barco de Valdeorras) }\end{array}$ & & $x$ & Xixón & 1937 \\
\hline $\begin{array}{l}\text { Longinos Rodríguez de la } \\
\text { Iglesia }\end{array}$ & & Regueiro (Boborás) & $x$ & & O Carballiño & \\
\hline Félix Salgado del Moral & & Castrelo do Miño & & $x$ & $\begin{array}{l}\text { Fóra de } \\
\text { Galicia }\end{array}$ & \\
\hline Francisco Sierra Rodríguez & 52 & Mosteiro (Xinzo) & $x$ & & Allariz & 1936 \\
\hline
\end{tabular}

\section{Outro profesorado}

\begin{tabular}{l|c|l|l|l|l|l}
\hline Manuel Gómez del Valle & 30 & Mestre & & X & Ourense & 1936 \\
\hline Gonzalo Martín March & 39 & $\begin{array}{l}\text { Mestre } \\
\text { (Gobernador civil Ourense) }\end{array}$ & X & Ourense & 1936 \\
\hline Eduardo Villot Canal & 28 & $\begin{array}{l}\text { Mestre } \\
\text { (Colexio Sueiro de Ourense) }\end{array}$ & X & & A Bola & 1936 \\
\hline Jacinto Santiago García & 42 & $\begin{array}{l}\text { Escola de Maxisterio } \\
\text { (Ourense) }\end{array}$ & X & & Montederramo & 1936 \\
\hline
\end{tabular}




\section{PONTEVEDRA}

\section{Maxisterio público en exercicio na provincia en xullo de 1936}

\begin{tabular}{|c|c|c|c|c|c|c|}
\hline \multirow{2}{*}{ Nome } & \multirow{2}{*}{ Idade } & \multirow{2}{*}{ Escola onde exercía } & \multicolumn{3}{|c|}{ Morte } & \multirow{2}{*}{ Ano } \\
\hline & & & $\mathbf{P}$ & $E$ & Concello & \\
\hline Segundo Abal Padín & 25 & Castrogudín (Vilagarcía) & & $x$ & Pontevedra & 1938 \\
\hline Germán Adrio Mañá & 48 & Pontevedra & & $x$ & Pontevedra & 1936 \\
\hline Juan Alonso Pérez & & Arbo & $x$ & & Cotovade & 1936 \\
\hline Joaquín A. Álvarez Álvarez & 43 & Pazos (Arbo) & & $x$ & Vigo & 1936 \\
\hline José Cortés Fernández & 39 & Goián (Tomiño) & & $x$ & Pontevedra & 1936 \\
\hline Victor Fraíz Villanueva & 50 & Coia (Vigo) & & $x$ & Vigo & 1937 \\
\hline Hipólito Gallego Camarero & 39 & Forcadela (Tomiño) & $x$ & & Mondariz & 1936 \\
\hline $\begin{array}{l}\text { Constantino Gómez } \\
\text { Rodríguez }\end{array}$ & 25 & Setecoros (Valga) & & $x$ & Pontevedra & 1937 \\
\hline Manuel Guillán Abalo & 34 & Moreixas (Lalín) & $\mathrm{x}$ & & A Estrada & 1936 \\
\hline Manuel Iglesias Filgueira & 31 & Xinzo (Ponteareas) & $x$ & & Pontevedra & 1936 \\
\hline $\begin{array}{l}\text { Manuel Jesús Juncal } \\
\text { Paredes }\end{array}$ & 38 & O Porriño & $x$ & & Vigo & 1937 \\
\hline José Meis Martínez & 42 & O Seixo (Marín) & & $x$ & Pontevedra & 1937 \\
\hline Antonio Mojón Vázquez & 21 & A Cañiza & & $x$ & Vigo & 1936 \\
\hline Eduardo Muíños Búa & 39 & Xeve (Pontevedra) & & $x$ & Pontevedra & 1936 \\
\hline Paulo Novás Souto & 36 & Pontevedra & & $x$ & Pontevedra & 1936 \\
\hline Manuel Pedreira Pazos & & Meavía (Forcarei) & $x$ & & & 1936 \\
\hline Jesús Eugenio Pérez Pérez & 39 & Achas (A Cañiza) & & $x$ & Vigo & 1936 \\
\hline Benigno Rey Pavón & 27 & Pontevedra & & $x$ & Pontevedra & 1936 \\
\hline Alfonso Rodrigo Méndez & 41 & Padriñán (Sanxenxo) & & & & 1936 \\
\hline Vicente Sáez Ruíz & & Coiro-Pósito (Cangas) & $x$ & & & 1936 \\
\hline Víctor Sánchez Martil & 38 & Coiro (Cangas) & & $x$ & Pontevedra & 1936 \\
\hline Apolinar Torres López & 41 & Vigo & & $x$ & Vigo & 1936 \\
\hline Víctor Trabazo Serapio & & Xill (Meaño) & $x$ & & & \\
\hline José Vázquez Grela & 50 & Nigrán & $x$ & & Nigrán & 1936 \\
\hline Nicanor Villar Martínez & 36 & Mazaricos & $x$ & & \begin{tabular}{|l} 
Caldas de \\
Reis
\end{tabular} & 1936 \\
\hline
\end{tabular}




\begin{tabular}{|c|c|c|c|c|c|}
\hline \multicolumn{6}{|c|}{ Outro profesorado } \\
\hline Manuel Barros Pérez & & Mestre (Vilagarcía) & Cárcere & $\begin{array}{l}\text { Illa San } \\
\text { Simón }\end{array}$ & \\
\hline José María Fernández Inclán & 27 & Mestre (Asturias) & $X$ & A Guarda & 1938 \\
\hline Manuel de la Rica Calderón & 68 & Mestre & Cárcere & A Guarda & 1940 \\
\hline Valentín Soriano Moreno & 63 & Mestre & Cárcere & $\begin{array}{l}\text { Illa San } \\
\text { Simón }\end{array}$ & \\
\hline José Caldas & & Escola de Traballo (Vigo) & & & \\
\hline
\end{tabular}

